\title{
Centimeter-scale hole diffusion and its application in organic light-emitting diodes for reducing efficiency roll-off and enhancing operation lifetime
}

Shihao Liu

City University of Hong Kong https://orcid.org/0000-0002-0645-5319

Chunxiu Zang

Jilin University

Xiang Zhang

Jilin University

Dong Shen

City University of Hong Kong

Hongwei Yu

Jilin University

Menglin Li

City University of Hong Kong

Hui Wang

City University of Hong Kong

Zhongqiu Tong

City University of Hong Kong

Ming-Fai Lo

Center of Super-Diamond and Advanced Films (COSDAF) and Department of Physics and Materials

Science, City University of Hong Kong

Letian Zhang

Jilin University

Wenfa Xie

Jilin University https://orcid.org/0000-0003-0912-8046

Chun-Sing Lee ( $\square$ apcslee@cityu.edu.hk)

City University of Hong Kong https://orcid.org/0000-0001-6557-453X

\section{Article}

Keywords: hole lateral diffusion, array electrode, carrier accumulation, triplet-polaron annihilation, low efficiency roll-off 
Posted Date: March 10th, 2021

DOI: https://doi.org/10.21203/rs.3.rs-289737/v1

License: (c) (i) This work is licensed under a Creative Commons Attribution 4.0 International License. Read Full License 


\title{
Centimeter-scale hole diffusion and its application in organic light-emitting
} diodes for reducing efficiency roll-off and enhancing operation lifetime

Shihao Liu ${ }^{1,2}$, Xiang Zhang ${ }^{1}$, Chunxiu Zang ${ }^{1}$, Dong Shen ${ }^{2}$, Hongwei $\mathrm{Yu}^{1}$, Menglin $\mathrm{Li}^{3}$, Hui Wang $^{2}$, Zhongqiu Tong ${ }^{2}$, Ming-Fai Lo $^{2}$, Letian Zhang ${ }^{1}$, Wenfa Xie ${ }^{1 *}$ and Chun-Sing Lee ${ }^{2 *}$ ${ }^{1}$ State key Laboratory of Integrated Optoelectronics, College of Electronics Science and Engineering, Jilin University, Changchun, 130012, People's Republic of China

${ }^{2}$ Center of Super-Diamond and Advanced Films (COSDAF) and Department of Chemistry, City University of Hong Kong, Hong Kong SAR, 999077, People's Republic of China ${ }^{3}$ Department of Materials Science and Engineering, City University of Hong Kong, Hong Kong SAR, 999077, People's Republic of China *e-mail: xiewf@jlu.edu.cn, apcslee@cityu.edu.hk

\begin{abstract}
We found that hole diffusion in a centimeter-scale can be achieved in a PEDOT:PSS layer via composition and interface engineering. This ultralong distance hole diffusion enables substantially enhanced hole diffusion current in the lateral direction perpendicular to the applied electric field in typical organic optoelectronic devices. By introducing this lateral-holediffusion layer (LHDL) at the anode side of organic light-emitting diodes (OLEDs), both reduced efficiency roll-off and enhanced operation stability are demonstrated. In conventional OLEDs, balance in electron and hole currents is typically achieved by leakage of the major carrier through the devices or by accumulation of the major carrier inside the devices. Both of these are known to reduce performances leading to efficiency roll-off at high currents, reduction of operation stability due to exciton-polaron annihilation etc. The application of the LHDL provides a new strategy for current balancing with much reduced harmful effects from the previous two approaches. For example, by incorporating the diffusion layer in a white phosphorescent OLED, $94 \%$ of its maximum efficiency can be maintained even at a brightness of $10000 \mathrm{~cd} / \mathrm{cm}^{2}$. At a high brightness of $30000 \mathrm{~cd} / \mathrm{cm}^{2}$, the OLED maintains a record high
\end{abstract}


external quantum efficiency of $13.9 \%$ without using any optical photon extraction layer. The OLED also show 5.5 times improvements in operation lifetime over the device without the diffusion layer. This study shows that centimeter-scale hole diffusion can be achieved in organic semiconductors and generally applied for enhancing efficiency and stability of OLEDs.

\section{Keywords}

hole lateral diffusion, array electrode, carrier accumulation, triplet-polaron annihilation, low efficiency roll-off

\section{TOC}

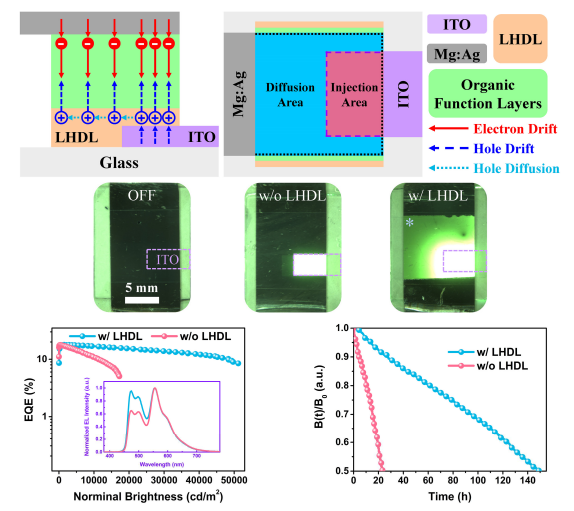


While full-color organic light-emitting diode (OLED) displays have been widely commercialized in the past decade, commercial products of OLED solid state lighting are still rare. One important reason is that OLEDs, especially high efficiency phosphorescent OLEDs, do have a common disadvantage that their efficiencies show significant roll-off at high brightness ${ }^{[1-9]}$. It is thus important to address this issue for promoting OLEDs' lighting applications. The "efficiency roll-off" issue is caused by different non-linear and complicated processes related to organic semiconductors' intrinsic properties, such as charge imbalance, bimolecular quenching processes and exciton dissociation induced by heat or electric field ${ }^{[9-}$ 11]. For organic semiconductors, hole drifting mobilities in hole-transporting materials are typically several orders of magnitude higher than electron mobilities in electron-transporting materials $[9,10,12]$. It is generally considered that such mobilities differences would lead to unmatched electron and hole currents. In fact, Fave et al have shown that there are two possible scenarios in typical OLEDs ${ }^{[13]}$. In the first situation, after electron-hole recombination, the surplus carriers (typically hole) will flow through the whole devices. These leakage currents not only waste energy without generating light, it can also decrease operation lifetime. For example, Aziz et al have shown that leakage of hole current can cause degradation of Alq3 in a prototypical NBP/Alq3 device ${ }^{[14]}$. In many high efficiency devices, hole-blocking layers are thus inserted to suppress the hole leakage current. This leads to the second scenario that excessive holes would accumulate inside the devices leading to built-in electric fields which would suppress the hole current and finally achieve balanced electron and hole currents. However, these accumulated holes can form polarons and can quench excitons via excitonpolaron annihilation leading to decreased efficiency and operation lifetime ${ }^{[15-24]}$. It can be seen that in either scenario, both efficiency and operation lifetime are harmfully affected. The key question here is that before we can develop materials with matching electron and hole mobilities is there a third scenario beyond the above two described by Fave et al? 
In this work, we show that a third scenario is possible via introducing current flow in the lateral direction (i.e. perpendicular to the applied electric field). So far almost all studies of OLEDs are mainly focused on the carrier dynamics along the direction of electric field; namely the drifting of carriers perpendicular to the planar devices ${ }^{[25-27]}$. However, charge carriers can also diffuse in all directions, including perpendicular to the electric field, as carrier "diffusion" is driven by concentration gradient and is independent of the electric field ${ }^{[28]}$. Recently, Leo et al for the first time constructed an electron lateral transport layer with a conductivity of $4 \mathrm{~S} / \mathrm{cm}$ by doping $\mathrm{C}_{60}$ with $16 \mathrm{wt} \%$ of an electron donor material tetrakis(1,3,4,6,7,8-hexahydro2Hpyrimido [1,2a] pyrimidinato)ditungsten (II) $\left[\mathrm{W}_{2}(\mathrm{hpp})_{4}\right]$, and observed emission spreading of $\sim 300 \mu \mathrm{m}$ outside the anode-cathode overlapping region ${ }^{[29,30]}$. Forrest et al later showed a breakthrough that lateral electron diffusion in centimeter scale can be achieved in a doped $\mathrm{C}_{60}$ channel at room temperature ${ }^{[31]}$. This centimeter-scaled electron diffusion is of critical importance for understanding fundamental physics in organic optoelectronic devices and can be applied for making charge coupled devices ${ }^{[32]}$. On the other hand, such long-range diffusion for hole has not be reported.

In this work, we demonstrated that hole diffusion in centimeter length scale can be achieved in PEDOT:PSS layers via composition and interface engineering. Such ultralong scale hole diffusion is further exploited to address the issue of efficiency roll-off in OLEDs. It was found that lateral hole diffusion beyond the cathode-anode overlapping region can redistribute carriers and induce additional electron injection from the cathode/organic interface. This leads to effective reduction of hole accumulation inside the device and thus reduces efficiency roll-off. We demonstrate this by incorporating a lateral-hole-diffusion layer (LHDL) in a white phosphorescent OLED. 94\% of its maximum EQE (EQEmax) can be maintained even at a high brightness of $10000 \mathrm{~cd} / \mathrm{cm}^{2}$. The device also shows high EQE of $13.9 \%$ at brightness of 30000 $\mathrm{cd} / \mathrm{cm}^{2}$. To the best of our knowledge, this device shows one of the highest EQE at brightness 
beyond $30000 \mathrm{~cd} / \mathrm{cm}^{2}{ }^{[33-36]}$. The device also shows over 5 times of operation lifetime enhancement comparing with the corresponding device without the LHDL.

\section{Properties of conventional devices without lateral-hole-diffusion layer}

$\mathbf{a}$

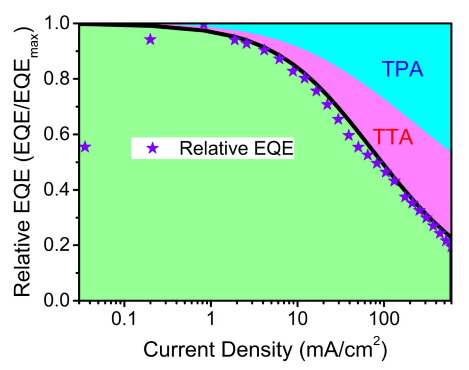

d

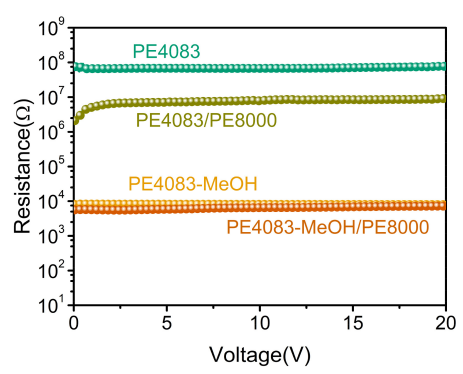

b

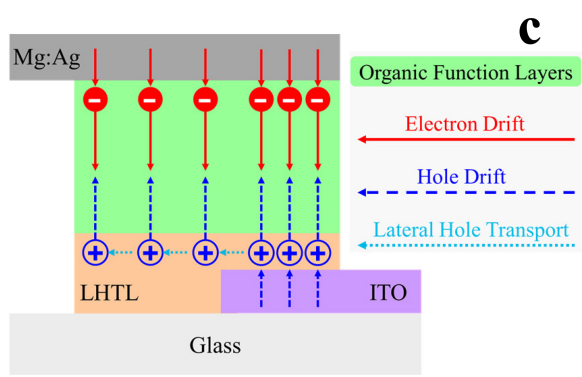

e

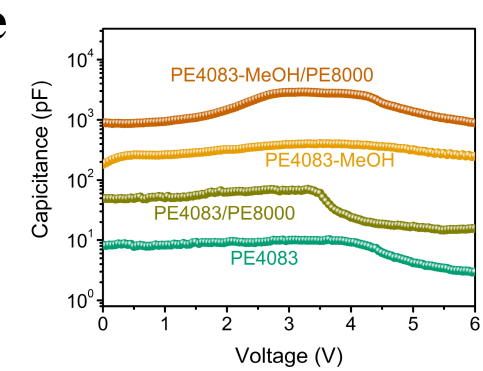

$\mathbf{f}$
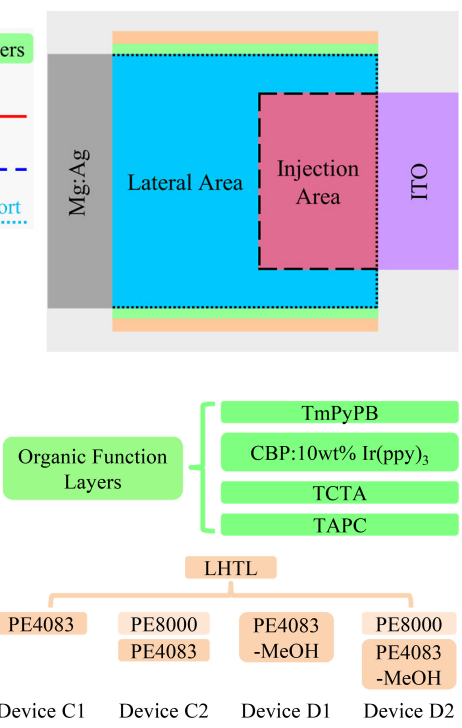

$\mathbf{g}$
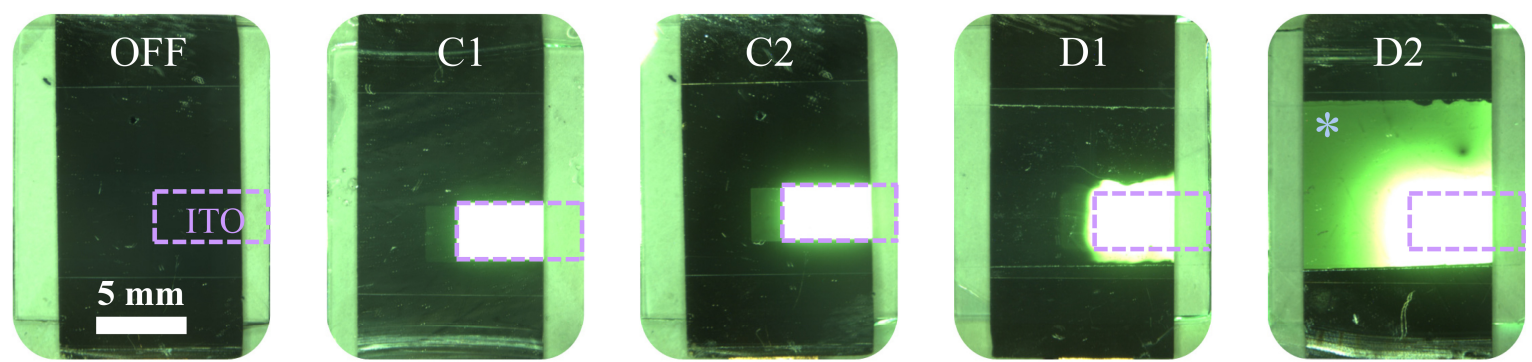

Figure 1. (a) Theoretically calculated contributions of TTA and TPA to EQE loss in device C1 as a function of current density. Experimentally measured relative EQE is shown as symbol $\star$; (b) Side-view schematic diagram of an OLED with a lateral-hole-transport layer, and (c) its top view. (d) Lateral resistance and (e) capacitance characteristics of different types of PEDOT:PSS layers. (f) Devices structures of devices C1, C2, D1 and D2. (g) Top-view images of these devices under $6 \mathrm{~V}$.

Firstly, a green phosphorescent OLED with a conventional device structure of ITO/PEDOT:PSS $\quad(30 \quad \mathrm{~nm}) /$ TAPC $\quad(30 \quad \mathrm{~nm}) /$ TCTA $\quad(5 \quad \mathrm{~nm}) / \mathrm{CBP}: 10 \mathrm{wt} \% \quad \operatorname{Ir}(\text { ppy })_{3}$

$\mathrm{nm}) / \mathrm{TmPyPhB}(50 \mathrm{~nm}) / \mathrm{LiF}(1 \mathrm{~nm}) / \mathrm{Mg}: 10 \mathrm{wt} \% \mathrm{Ag}(100 \mathrm{~nm})$ was fabricated and called device C1. Details of the PEDOT:PSS layer and full names of the compounds are given in the experimental section. Characteristics of device $\mathrm{C} 1$ are given in supporting information (Figure S1 and Table S1). Relative EQE with respect to the maximum EQE (EQE $\max$ ) of the device is 
shown with $\star$ symbols in Figure 1a. It can be seen that EQE of the device decreases considerably when the current density is higher than $1 \mathrm{~mA} / \mathrm{cm}^{2}$. Only about $20 \%$ of the $\mathrm{EQE}_{\max }$ can be maintained when the current density is $600 \mathrm{~mA} / \mathrm{cm}^{2}$. The significant EQE loss at high current densities in typical phosphorescent OLEDs have been attributed to triplet-polaron annihilation (TPA) and triplet-triplet annihilation (TTA) ${ }^{[9,10,18]}$. Following the approach of Leo et al ${ }^{[9]}$ and with refer to the parameters (Figure S2a and Table S2), we proceed to determine their relative contributions by analyzing densities of triplets $\left(n_{T}\right)$ and polaron $\left(n_{P}\right)$ at steady state. Calculated relative EQE losses due to TTA and TPA are respectively shown as pink and cyan areas in Figure 1a. Calculated densities of triplet and polaron at different current densities are given in Figure $\mathrm{S} 2 \mathrm{~b}$.

As shown in Figure 1a, TPA dominates the quenching process across the whole current range. This phenomenon can be attributed to excessive hole accumulation caused by the unmatched hole and electron transporting abilities of the organic layers. To confirm this, we prepare a holeonly (C1h) and an electron-only (C1e) devices by modifying the structure of device $\mathrm{C} 1$ (Figure S3). Figure S4a shows that current density of device C1h is much higher than that of device C1e. From this result, it can be reasonably considered that the hole and electron transporting abilities of device $\mathrm{C} 1$ are highly unmatched (hole dominate). We then measured the capacitancevoltage characteristic of device C1 (Figure S4b). It can be seen that upon carrier injection (at $2.8 \mathrm{~V}$, point a'), capacitance of device $\mathrm{C} 1$ shows an initial increase as voltage increases. This is attributed mostly to holes accumulation in the emitting layer based on the discussion in Supplementary Note 1.

Using the "Marburg model" (Figure S5a) ${ }^{[13,37]}$, the reason of carrier accumulation in device $\mathrm{C} 1$ is explored in Supplementary Note 2. In the operating hole-dominant device C1 (Figure $\mathrm{S} 5 \mathrm{~b})$, the recombination current $I_{R}$ can be expressed as:

$I_{R}=I_{h}-I_{h^{\prime}}-\frac{d Q_{h}}{d t}=I_{e}$ 
where, $I_{h}$ and $I_{e}$ are respectively the injected hole and electron currents at the electrode/organic interfaces. $I_{h}$, is the leakage hole current beyond the recombination zone. $Q_{h}$ is the accumulated hole charges inside the device. Due to efficient hole blocking abilities of TmPyPB, $I_{h}{ }^{\prime}$ can be approximated to zero for device $\mathrm{C} 1$. Thus, the balance of hole and electron currents is mainly achieved via the term $\mathrm{d} Q_{h} / \mathrm{dt}$. Upon carrier injection (Figure S5b), $Q_{h}$ increase steadily, such that internal potential distribution will be affected. The potential difference $\left(E_{h}\right)$ across hole transport region will decrease, leading to a decreased $I_{h}$. Until finally, enough $Q_{h}$ is accumulated (ie. $\left.\mathrm{d} Q_{\mathrm{h}} / \mathrm{dt}=0\right)$ and lead to a dynamic equilibrium between hole and electron currents $\left(I_{h}=I_{e}\right)$. Under this dynamic equilibrium, internal electric field distribution is highly uneven. For example, Fave et al showed that $E_{e}$ can be over 3 times higher than $E_{h}{ }^{[13]}$. Given that the electric field for typical OLEDs is high $\left(>10^{7} \mathrm{~V} / \mathrm{m}\right)$, a considerable number of holes would accumulate in the device interior $\left(10^{11} \sim 10^{12} \mathrm{~cm}^{-2}\right.$, with refer to our result as shown in Figure S4b). The accumulated holes finally lead to serious TPA in device C1 (Figure 1a).

\section{Design of the Lateral-hole-diffusion Layer}

To address the issue of excessive hole accumulation, we proposed to introduce a lateral hole current $I_{L}$ by inserting a lateral hole transport layer (LHTL) between ITO and organic function layers, as shown in Figure $1 \mathrm{~b}$ and $1 \mathrm{c}$. The $I_{L}$ is anticipated to generate an additional electric field helping to extract electrons from the cathode side. As discussed in Supplementary Note 3, we show that after introducing the LHTL, it is possible to achieve hole-electron current balance without involving (or with much reduced) hole accumulation. However, Supplementary Note 3 also shows that the LHTL-induced current balance only works under the assumption that the lateral current $I_{L}$ is obtained via diffusion from the LHTL layer at the anode-cathode overlapping region instead of injection from the ITO. Further evidences supporting this assumption are provided in later parts of the paper.

PEDOT:PSS is exploited for applications as the lateral transport layer as its conductivity can be changed over a wide range by adjusting its composition and configuration (Figure S6). 
Figure 1d shows lateral resistivities (Figure S7) of different types of PEDOT:PSS films, including PE4083 (PEDOT:PSS Clevios ${ }^{\mathrm{TM}}$ P VP AI 4083, $30 \mathrm{~nm}$ ), PE4083/PE8000 bilayer (PEDOT:PSS Clevios' ${ }^{\mathrm{TM}}$ P VP: AI 4083, 30 nm/CH 8000, 30 nm), PE4083-MeOH (methanoltreated PE4083), PE4083-MeOH/PE8000. Details on preparation of these PEDOT:PSS films are given in the Experimental Section. As shown in Figure 1d, untreated PEDOT:PSS films (PE4083, PE4083/PE8000) have higher resistivities, while treatment with methanol (MeOH) can substantially decrease their resistivities. It is interesting to note that PEDOT:PSS has simultaneously high conductivity and capacitance (Figure 1d, 1e). In fact, holes can be stored at the interface between the conducting PEDOT rich phase and the insulating PSS rich phase [38, 39]. Figure 1e shows that the capacitance of PEDOT:PSS can be increased over two orders of magnitudes upon treatments.

Devices C1, C2, D1 and D2 were then prepared using respectively PE4083, PE4083/PE8000, PE4083-MeOH and PE4083-MeOH/PE8000 as shown in Figure 1f. Figure 1g shows images of the devices operating at $6 \mathrm{~V}$. It can be seen that the emitting area in devices $\mathrm{C} 1$ and $\mathrm{C} 2$ are limited to the cathode-ITO overlapping region. Apparent emission spreading out of the cathodeITO overlapping region can be observed in devices D1 and D2. Apparently, the conductivities of PE4083 and PE4083/PE8000 layers are not high enough to allow adequate lateral hole current $I_{L}$ such that devices $\mathrm{C} 1$ and $\mathrm{C} 2$ behave as conventional devices with no observable emission spreading. The high conductivity of PE4083-MeOH and PE4083-MeOH/PE8000 in devices D1 and D2 enable enhanced lateral hole current $I_{L}$ and thus emission spreading. In particular, emission can still be obtained in device D2 from regions of centimeter-scale away from the cathode-ITO overlapping region. It can be noted that device D2 shows much wider emission spreading than device D1 (Figure 1g), although their PEDOT:PSS layers have similar lateral conductivities ( $\sim \mathrm{S} / \mathrm{cm}$, Figure 1d). This suggests that the capacitance (Figure 1e) of the PEDOT:PSS layer might have influences on the lateral current $I_{L}$. Thus, we further measure the lateral impedance spectra of the PEDOT:PSS layers in devices C2 and D2 with the 
configuration as shown in Figure S7. It can be clearly seen that the PH4083-MeOH/PH8000 film behaves as a Warburg short element (Figure S8a), while the PH4083/PH8000 film behaves as a Warburg open element (Figure S8b). This indicates that hole transport in the former (latter) layer belongs to a finite-length diffusion with transmissive (reflective) boundary ${ }^{[40]}$. The hole diffusivity, D, of the PE4083-MeOH/PE8000 film is then estimated as $\sim 3 \times 10^{-3} \mathrm{~cm}^{2} / \mathrm{s}$ (Supplementary Note 4$)$, which is nearly two orders of magnitude higher than that $\left(\sim 3 \times 10^{-5}\right.$ $\mathrm{cm}^{2} / \mathrm{s}$ ) of the PE4083/PE8000 film. We can thus reasonably consider that lateral carrier transport in the PEDOT:PSS layers belongs to hole diffusion behavior as a result of the combination of their high conductivity and capacitance properties. For this reason, these PEDOT:PSS layers are hereafter referred as lateral hole diffusion layer (LHDL).

We then measured transient electroluminescence (EL) of devices C2 and D2 from a far field such that emissions both within and outside the cathode-ITO overlapping region can be collected (Figure 2a). It can be seen that the transient EL in device C2 matches well to the electrical pulse showing little delay. Interestingly, the transient EL in device D2 shows two rising stages. The initial rise $(<10 \mu \mathrm{s})$ is nearly identical to that of device $\mathrm{C} 2$ (see inset of Figure 2a). The delayed rise is much slower and emerges when device C2 maintains a steady state. Transient EL from device D2 was then measured from the spot marked as with “*” in Figure $1 \mathrm{~g}$ which is about $0.57 \mathrm{~cm}$ outside the cathode-ITO overlapping region using a longer electrical pulse of $480 \mu \mathrm{s}$ (Figure S9). It is interesting that no appreciable EL signal can be observed before $90 \mu$ s (see magnified region in the inset of Figure S9). This suggests that emission from this spot requires diffusion and accumulation of carriers from a long distance away. Thus, the initial and delayed emissions shown in Figure 2a corresponds to emissions within and outside the cathode-ITO overlapping region, respectively. However, transient current characteristics (Figure 2b) show that the external currents of devices C2 and D2 keep unchanged after initial charging process (i.e. after $10 \mu \mathrm{s}$ ) for activating the OLED. This suggests that the $I_{L}$ for driving 
$\mathbf{a}$

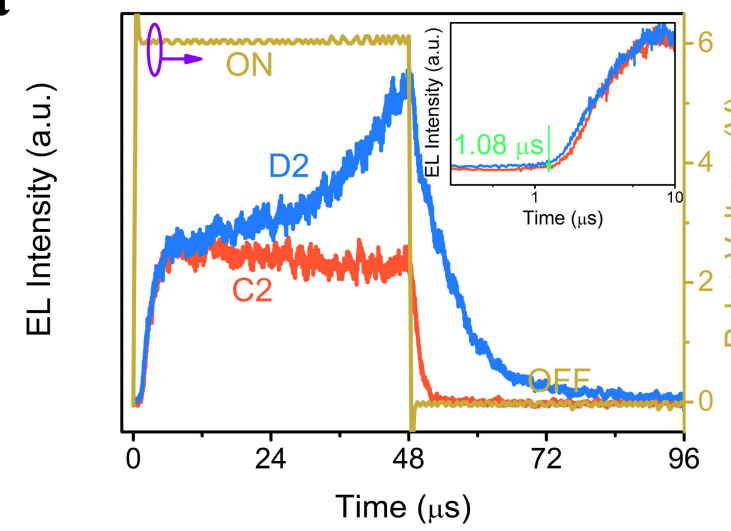

C

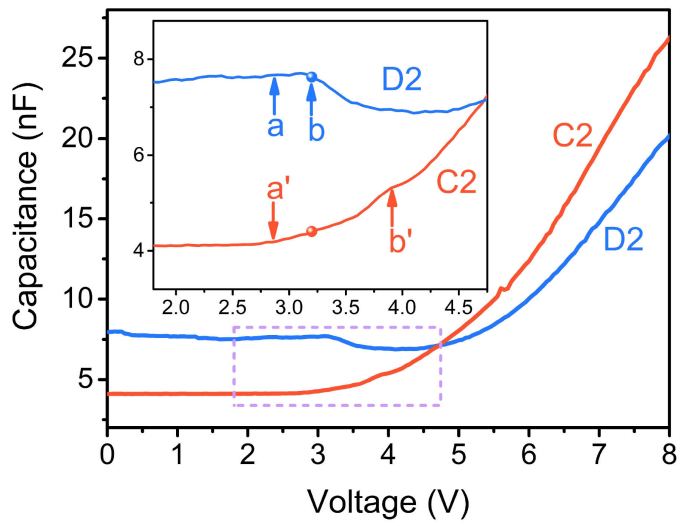

$\mathbf{e}$

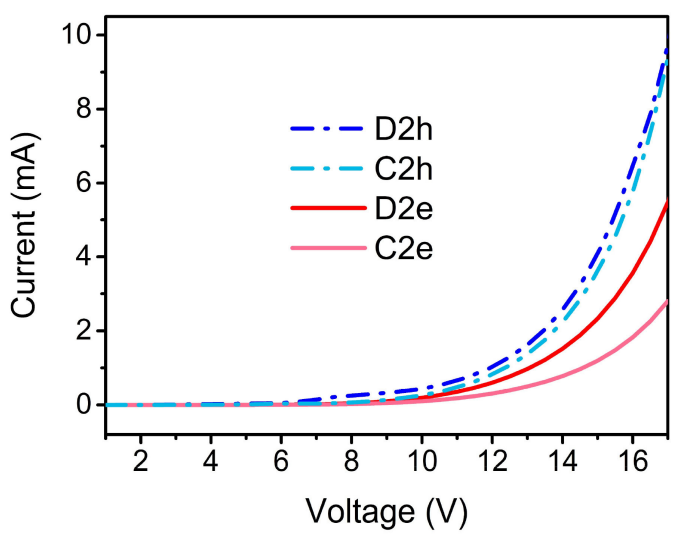

b

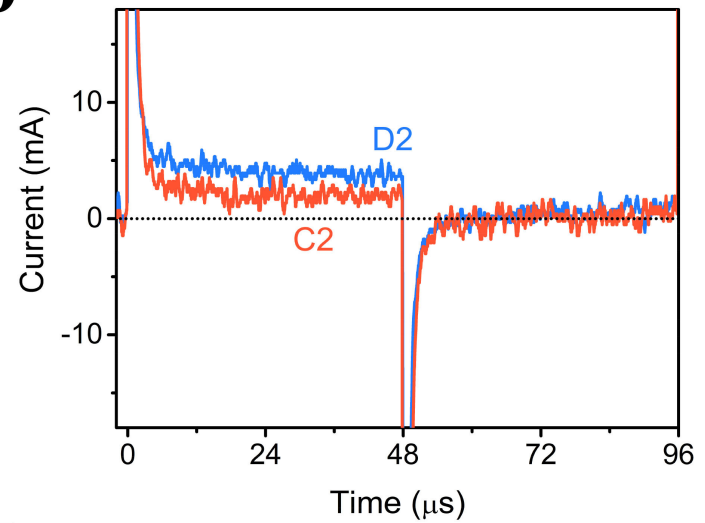

d

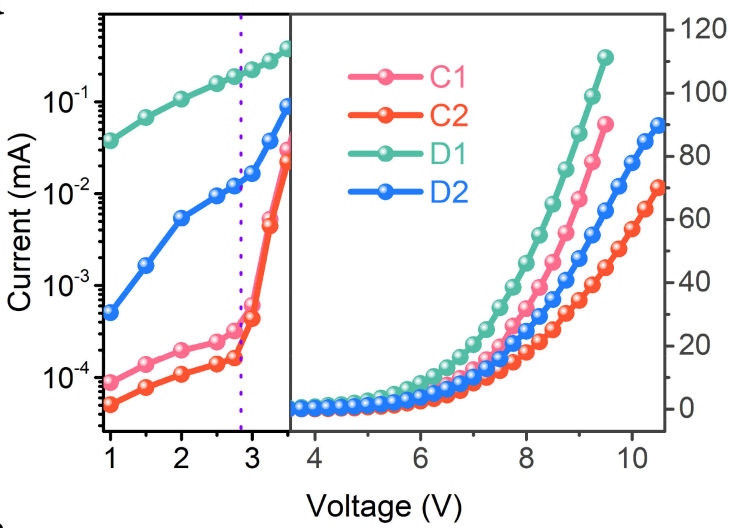

f

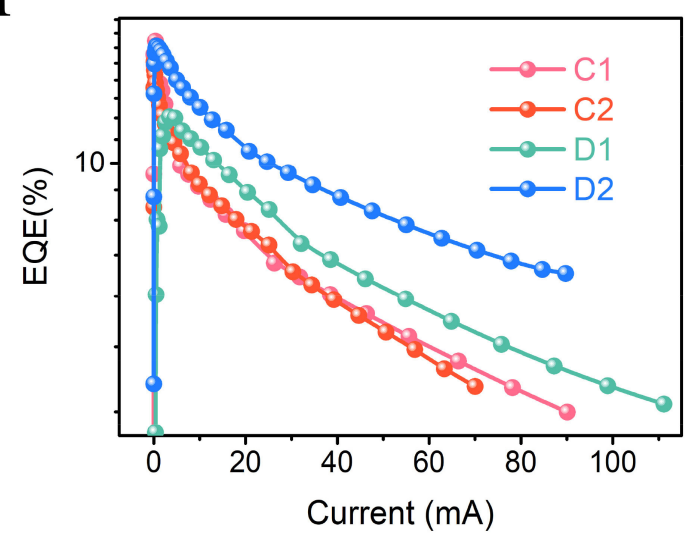

Figure 2. (a) Transient EL responses of devices C2 and D2, and (b) their transient current characteristics; the voltage pulse width and heights were respectively $48 \mu \mathrm{s}$ and $6 \mathrm{~V}$. (c) Capacitance-voltage characteristics of devices C2 and D2. (d) Current-voltage characteristics of devices C1, C2, D1 and D2. (e) Current-voltage characteristics of single carrier devices for device $\mathrm{C} 2$ and $\mathrm{D} 2$ (-e and -h represents electron-only device and hole-only device, respectively). (f) EQE-current characteristics of devices C1, C2, D1 and D2.

the delayed EL rise is not directly injected from the ITO anode. The delayed EL can be more reasonably explained by lateral hole diffusion in the PEDOT:PSS layer driven by the hole concentration gradient. When the bias is first applied, carrier movement will be dominantly controlled by the vertical electric field. There will be little lateral current $I_{L}$ as there is no 
apparent carrier density gradient initially. The PEDOT:PSS will be steadily charged up with an increasing hole concentration. Upon fully charged, there will be a large enough lateral hole concentration gradient driving a lateral hole current $I_{L}$. The laterally diffuse holes would then induce additional electron injection from the cathode giving rise to the delayed EL. With this understanding, the hole-electron current balance can be achieved via lateral hole diffusion (as discussed in Supplementary Note 3):

$$
I_{h}=I_{e}+I_{L}=I_{e}-q D A \frac{d \rho_{L H D L}}{d x}
$$

The term $-q D A \frac{d \rho_{L H D L}}{d x}$ refers to lateral hole diffusion, and $\mathrm{A}$ is the longitudinal cross-section area of the LHDL at the border (dash line in Figure 1c) of the ITO-cathode overlapping region.

To further confirm this, capacitance-voltage characteristics of the devices and currentvoltage characteristics of their single-carrier devices (Figure S10) were measured. As shown in Figure 2c and S11, devices D1 and D2 show much higher geometric capacitance than devices $\mathrm{C} 1$ and $\mathrm{C} 2$. This is due to highly improved carrier storage ability of the PEDOT:PSS layers upon $\mathrm{MeOH}$ treatment (Figure S12 and Supplementary Note 5). After carrier injection (point $\mathrm{a} / \mathrm{a}$ ' in Figures 2c and S11, and dotted line in Figure 2d), devices C1 and C2 show capacitance increases from point a' to point b', corresponding to carrier accumulation in the emitting layer ${ }^{[41]}$. Interestingly, the capacitance changes in devices D1 (Figure S11) and D2 (Figure 2c) differ considerably from those of devices $\mathrm{C} 1$ and $\mathrm{C} 2$. It can be seen that upon turning on (at $3.1 \mathrm{~V}$, blue sphere/point b), capacitances of devices D1 and D2 show initial decreases as voltage increases. This gives an evidence that current balance in these devices is not solely achieved via carrier accumulation $\left(I_{R}=I_{h}-I_{h^{\prime}}-\frac{d Q_{h}}{d t}=I_{e}\right)$, and the lateral current $I_{L}$ should work for achieving current balance $\left(I_{h}=I_{e}+I_{L}\right)$. Furthermore, the lateral diffusion of hole makes it possible for achieving current balance without suppressing hole current. As shown in Figure 2d, the current of device D2 (D1) is much higher than that of device C2 (C1) under high bias voltages. According to the voltage-current characteristics (Figure 2e and S13) of single carrier 
devices, the current improvement of devices D2 and D1 is mainly attributed to the improvement in electron current (see $I_{e L}$ in Figure S5c).

Results from the device characteristics also support the influence of the lateral current $I_{L}$. We can note that performances of devices D2 and D1 are enhanced at high current (Figure S1). As current increases, device D2 (D1) shows much less EQE roll-off (Figure 2f) than device C2 (C1). A brightness enhancement within the ITO-cathode overlapping region of devices D2 and D1 is also observed (Figure S14). These results support the reduction of TPA within and outside the ITO-cathode overlapping region of devices D2 and D1.

\section{Working mechanism of an OLED with a LHDL}
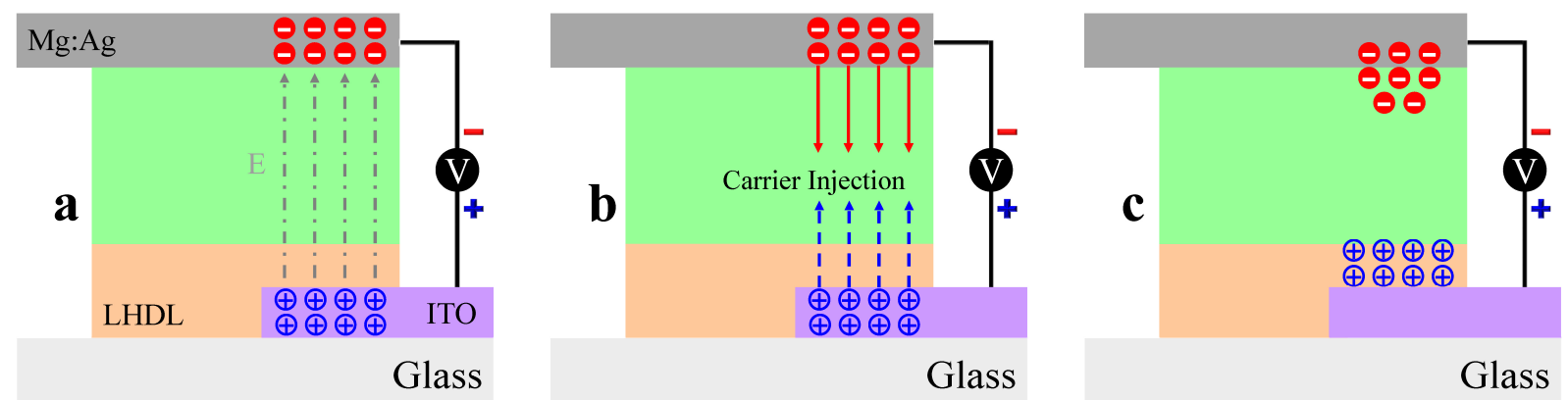

Glass

Glass

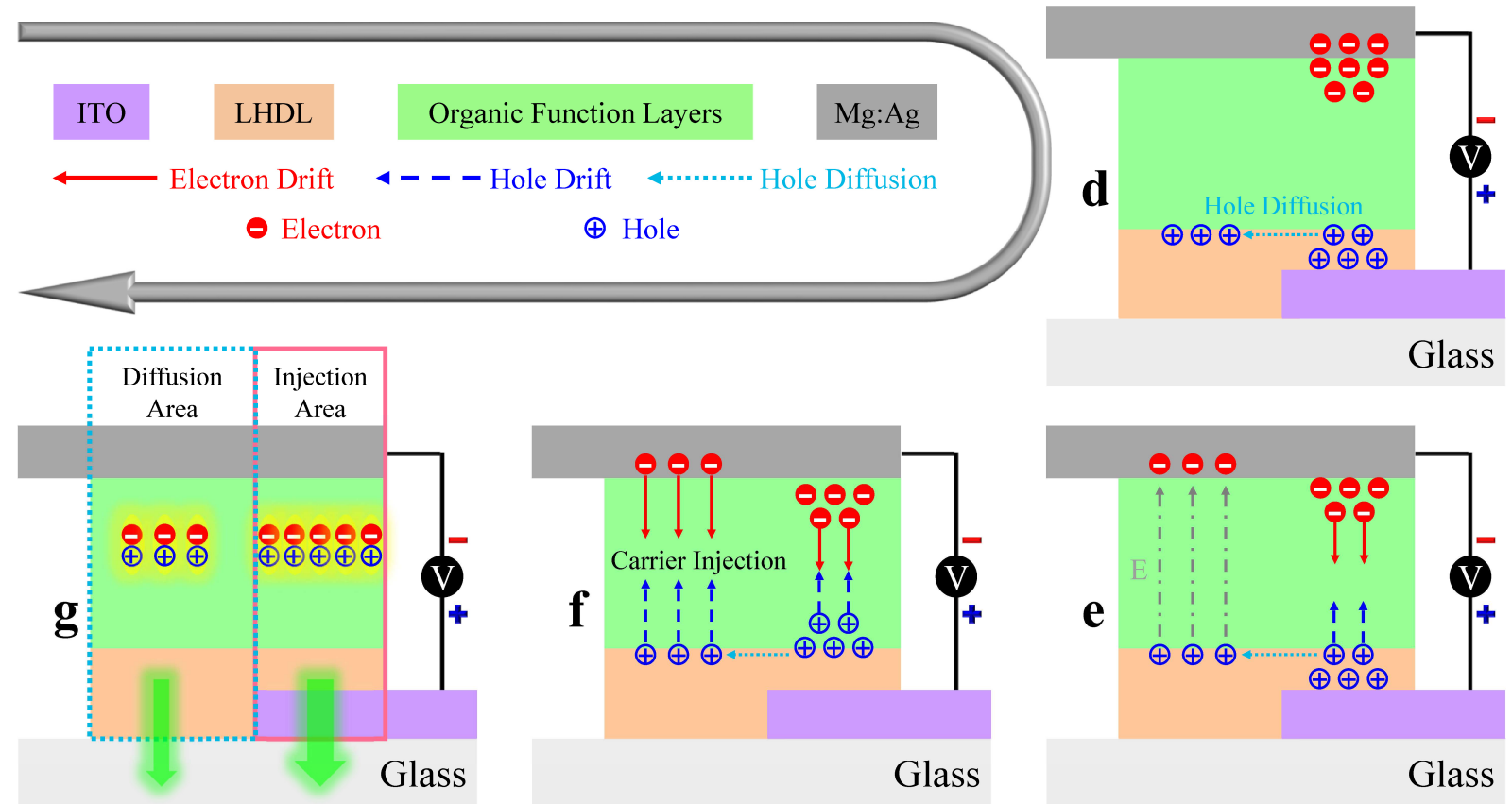

Figure 3. A working mechanism of an OLED with lateral hole diffusion. (a) Applying bias voltage across the anode and cathode. (b) Carrier injection in injection area. (c) Asymmetric hole and electron transport abilities. (d) Holes lateral diffusion. (e) Electrical field formation in diffusion area (i.e. area outside ITO-cathode overlapping region). (f) Carrier redistribution and 
injection in diffusion area. (g) Exciton recombination radiation.

As noted above, a working mechanism of the OLEDs with LHDL is proposed and shown in

Figure 3. Compared to electrons, holes are usually more easily injected into the organic layer from anode upon biasing. Due to the highly conductive and capacitive LHDL, excess holes can laterally diffuse outside of the ITO-cathode overlapping region. These diffused holes would then generate an electric field to redistribute electron distribution and assist electron injection from the top cathode. This can explain the observation that device D2 has a much-enhanced electron current comparing to device C2 (Figure 2e). Finally, the diffused holes and induced electrons form excitons, and photons can be produced from regions both inside and outside of the ITO-cathode overlapping regions. Because of the additional emission from the diffusion area, the total luminescence of device D2 is much higher than that of device C2 (Figure S1b). Due to the reduced hole accumulation in the emitting layer, TPA inside the ITO-cathode overlapping region is also reduced and thus resulting in a brightness enhancement inside the nominal device region (Figure S14).

\section{OLEDs with lateral-hole-diffusion layers and ITO array anodes}

As shown in Figure S15, the emission intensity shows an exponential decay with the distance outside the ITO-cathode overlapping region. This agrees well with the assumption that the lateral current $I_{L}$ is due to diffusion from the PEDOT:PSS layer (Figure S16 and Supplementary Note 6). To achieve quasi-uniform emission, application of the hole lateral diffusion for OLEDs is then developed by using an array of ITO grid anode (Figure 4a). Width of and spacing between the ITO fingers are shown in Figure S17. Device with the structure of device D2 was then fabricated on substrates with the ITO arrays and labelled with device D2AA'. The impedance spectra (Figure S18) and infrared images (Figure S19) show that the use of long and thin ITO array will not result in increased ITO resistance nor serious thermal effect. This is due to parallel circuit characteristics of this type of ITO electrode. Figure $4 \mathrm{~b}$ shows image (upper) of operating device D2AA'. Region marked with green circle is shown as magnified image 
(lower). It can be seen that overlapping of spread-out emissions from neighboring ITO finger eventually smooth out the intensity modulation and the fringe pattern is no longer observable in device D2AA'.

$\mathbf{a}$

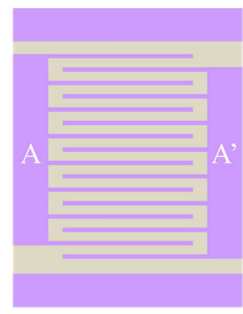
ITO $\mathrm{Mg}: \mathrm{Ag}$
LHDL
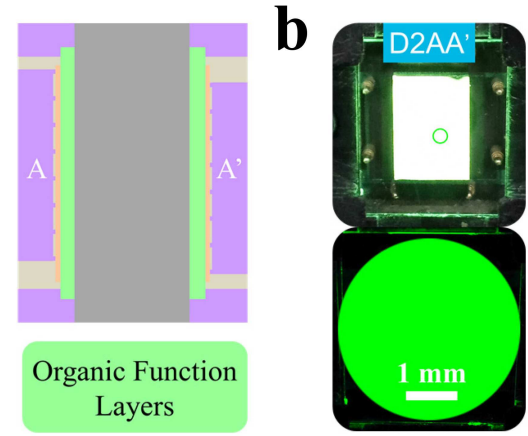

c
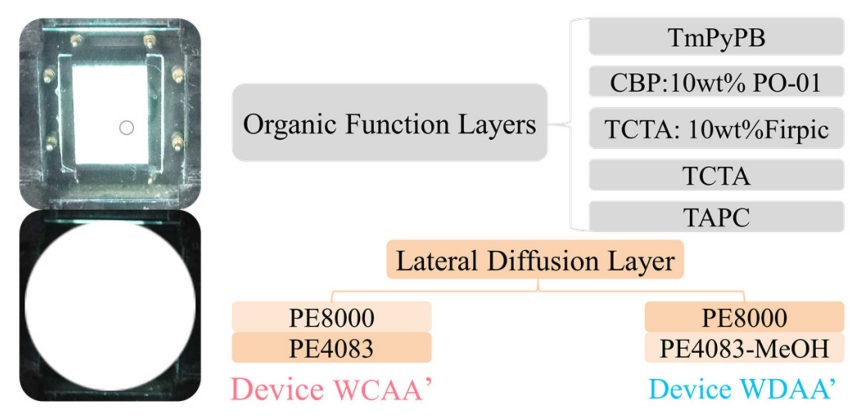
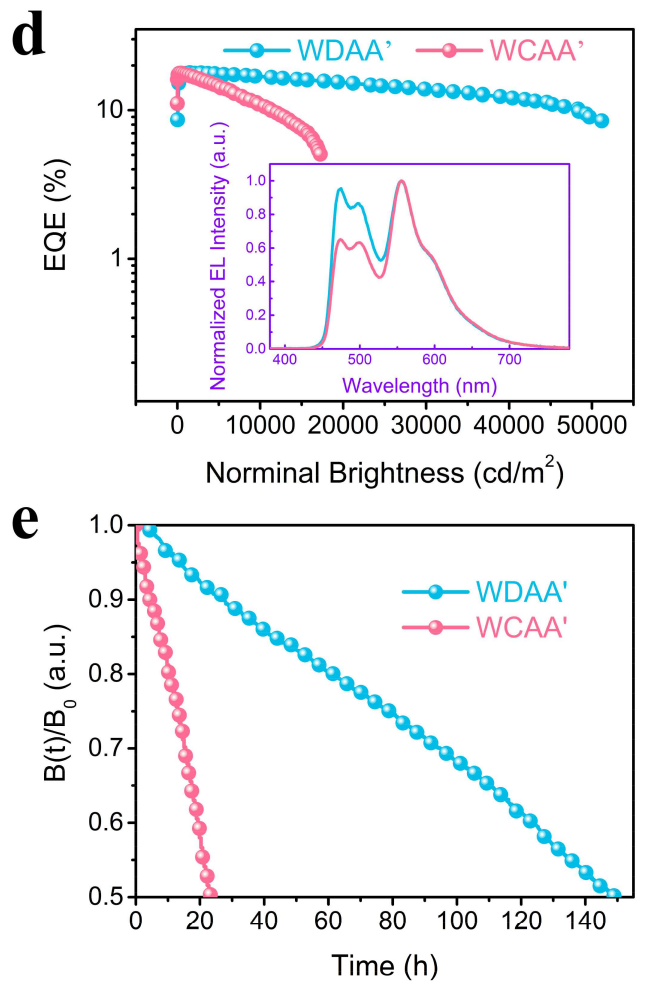

Figure 4. (a) Schematic diagram of the ITO grid array anode and the corresponding OLED. (b) Photographs (upper row) of operating device D2AA' with the ITO grid array anodes. Lower one shows magnified images from the green circles marked in the upper picture. (c) Photographs of white device WDAA' and structure details of white device WDAA' and WCAA'. (d) EQE-nominal brightness characteristics of the two white devices. Inset shows their EL spectra at $25 \mathrm{~mA} / \mathrm{cm}^{2}$. (e) Normalized brightness degradation $\mathrm{B}(\mathrm{t}) / \mathrm{B}_{0}\left(\mathrm{~B}_{0}=1000 \mathrm{~cd} / \mathrm{m}^{2}\right)$ of white devices WDAA' and WCAA'.

Finally, the diffusion layer and the ITO array electrode are applied in a white OLED (called device WDAA') employing Bis (3,5-difluoro-2-(2-pyridyl) phenyl-(2-carboxypyridyl) iridium (III) (Firpic) as a blue emitter and Iridium (III) bis (4-phenylthieno[3,2-c]pyridinato-N, $\mathrm{C}^{2}$ ) acetylacetonate (PO-01) as a yellow emitter (Figure 4c). For comparison, a corresponding white device WCAA' without the LHDL is also fabricated. Figure 4d and Figure S20 show EL performances of devices WDAA' and WCAA'. Apparently, device WDAA' with a LHDL possesses much higher EQE than device WCAA' with no LHDL under high current and brightness. At a nominal brightness of $10000 \mathrm{~cd} / \mathrm{m}^{2}$, the EQE of device WDAA' is $16.9 \%$, which 
is $94.4 \%$ of its maximum EQE (EQEmax) at $1495 \mathrm{~cd} / \mathrm{m}^{2}$. To the best of our knowledge, this is the smallest reported EQE roll-off among all white phosphorescent OLEDs at a high brightness of $10000 \mathrm{~cd} / \mathrm{m}^{2}$. For comparison, at $10000 \mathrm{~cd} / \mathrm{m}^{2}$, the EQE of device WCAA' is only $10.8 \%$ (61.0\% of its EQE $\max$ ). According to the EL spectra (Figure S21), shifting of emission spectra partly contribute to the extreme small efficiency roll-off in device WDAA'. Considering the higher proportion of yellow emission in WCAA' (see inset of Figure 4d), it is reasonably concluded that the lateral hole diffusion is the main cause of the efficiency enhancement in WDAA'. This strategy is not only specific to the devices above but represents a universal strategy that can be applied in different types of OLEDs (i.e. include blue, red phosphorescent devices, and TADF devices) for improving performances under high current (Figure S22 and Table S3).

More interestingly, the hole diffusion layer is also beneficial for improving operational lifetime of phosphorescent OLEDs. As shown in Figure 4e, the LT $_{50}$ lifetime (time to $50 \%$ of initial brightness of $1000 \mathrm{~cd} / \mathrm{m}^{2}$ ) of device WDAA' can attain 149 hours, corresponding to 5.5 times improvements compared to device WCAA'. Similar improvement can also be observed in blue device BDAA', compared with device BCAA' (Figure S23). Lifetime improvements of the blue and the white OLEDs with LHDLs should be attributed to the reduction of excitonpolaron annihilations in such devices, which are well known as a main reason for the short operational lifetime of phosphorescent OLEDs ${ }^{[24]}$.

In conclusion, centimeter-scale hole lateral diffusion behavior is observed in a PEDOT:PSS bilayer film which consists of a methanol treated PEDOT:PSS layer with high conductivity which form a highly capacitive interface with another untreated PEDOT:PSS layer. With this hole-diffusion layer, hole injected from the anode can diffused laterally in centimeter scale away from the anode. This process (1) generates spatial variation of carrier density for balancing carrier injection/transport in the normal emission region defined by the ITO-cathode overlapping area and (2) induces additional electron injection outside the ITO-cathode 
overlapping area. These not only lead to a higher luminescence resulting from the spread-out emission and also substantially reduce exciton loss due to polaron-exciton quenching due to the reduced excess hole density at the normal emission region. By combing this hole-diffusion layer with an ITO grid array anode, we demonstrate high performance phosphorescent OLEDs with record low EQE roll-off at high brightness and much improved device lifetime.

\section{Methods}

Materials: PEDOT:PSS (Clevios ${ }^{\mathrm{TM}}$ P VP AI 4083, Clevios ${ }^{\mathrm{TM}} \mathrm{P}$ VP $\mathrm{CH}$ 8000) and tris(2phenylpyridine)iridium(III) [Ir(ppy)3] were purchased from Xi'an Polymer Light Technology Corp. PEDOT:PSS (Clevios ${ }^{\mathrm{TM}}$ P VP AI 4083), di-[4-(N, N-di-p-tolyl-amino)phenyl]cyclohexane (TAPC), 4,4',4"-Tris(carbazol-9-yl) triphenylamine (TCTA), 4,4'Bis(carbazol-9-yl)biphenyl (CBP), bis (3,5-difluoro-2-(2-pyridyl)phenyl-(2-carboxypyridyl) iridium(III) (Firpic), bis (4-phenylthieno[3,2-c]pyridinato-N, ${ }^{2}{ }^{2}$ ) acetylaceto-nate iridium (III) (PO-01), 5,10-bis(4-(9H-carbazol-9-yl)-2,6-dimethylphenyl)-5,10-dihydroboranthrene (CzDBA), $\quad \operatorname{Bis}(2-m e t h y l d i b e n z o[f, \quad h] q u i n o x a l i n e)($ acetylacetonate)iridium(III) [Ir(MDQ)2(acac)], 1,3,5-Tri[(3-pyridyl)-phen-3-yl] benzene (TmPyPB) and LiF of OLED grade were purchased from Lumtech Corp. All of the materials were directly used without further purification.

OLED fabrication: Before using, the PEDOT:PSS aqueous solutions were filtered with 0.45 $\mu \mathrm{m}$ polyamide filters. ITO substrates $\left(25 \Omega \mathrm{sq}^{-1}\right)$ were cleaned by ultrasonic cleaning, dried at $120^{\circ} \mathrm{C}$, and then treated with air plasma for 5 min. Using the PEDOT:PSS (Clevios ${ }^{\mathrm{TM}} \mathrm{P}$ VP AI 4083 ) solution, PE4083 films were then spin-coated on the substrate at 5000 r.p.m. for $30 \mathrm{~s}$, followed by annealing at $120{ }^{\circ} \mathrm{C}$ for $10 \mathrm{~min}$. After that, some PE4083 films were soaked in methanol at $60{ }^{\circ} \mathrm{C}$ for $10 \mathrm{~min}$ and referred as $\mathrm{PE} 4083-\mathrm{MeOH}$ films. To obtained PE4083/PE8000 and PE4083-MeOH/PE8000 films, PE8000 films were then spin-coated on the PE4083 and PE4083-MeOH films at 5000 r.p.m. for 30 s using PEDOT:PSS (Clevios ${ }^{\mathrm{TM}} \mathrm{P}$ VP $\mathrm{CH} 8000$ ) solution, followed by annealing at $120^{\circ} \mathrm{C}$ for $10 \mathrm{~min}$. All the small molecular function 
layers and cathode were then deposited in turn on the PEDOT:PSS-coated substrates via thermal evaporation. Evaporating rates of the organic materials, $\mathrm{LiF}$, and $\mathrm{Mg}: \mathrm{Ag}$ are 1-2, 0.05-0.1 and 2-3 $\AA / \mathrm{s}$, respectively. The effective injection area was set to $3 \times 5 \mathrm{~mm}^{2}$ for devices $\mathrm{C} 1, \mathrm{C} 2, \mathrm{D} 1$ and D2, determined by the overlap between the Mg:Ag cathode and the ITO anode.

Device measurements and film characterization: EL performances of all OLEDs were measured in atmosphere at room temperature. Performance parameters not related to lightemitting area were measured with a goniophotometric measurement system (Otsuka Electronics, Japan) consisting of a Keithley 2400 source, a MCPD 9800 spectrometer, a light receiving fiber and an integration sphere. These parameters were synchronously determined with a measurement software from Otsuka Electronics, Japan. The brightness related to light-emitting area was measured with a brightness meter LS-110 Brightness Meter (Konica Minolta, Japan) targeted to a $\sim 0.4 \mathrm{~mm}$ diameter spot near the center of the devices (D1 and D2) which have an ITO-cathode overlapping area of $3 \times 5 \mathrm{~mm}^{2}$. Transient EL response was collected with a SPCST01 Fluorescence Spectrum Analyzer System (Beijing HOLITA, China). Impedance spectra and capacitance-voltage characteristics were measured with a ZAHNER IM6 and a HP44284A semiconductor analyzer.

\section{References}

1. $\mathrm{Wu}, \mathrm{T} .-\mathrm{L}$. et al. Diboron compound-based organic light-emitting diodes with high efficiency and reduced efficiency roll-off. Nat. Photonics 12, 235 (2018).

2. Zhang, D. et al. Versatile Indolocarbazole-Isomer Derivatives as Highly Emissive Emitters and Ideal Hosts for Thermally Activated Delayed Fluorescent OLEDs with Alleviated Efficiency Roll-Off. Adv. Mater. 30, 1705406 (2018).

3. Liu, H. et al. High-Performance Non-doped OLEDs with Nearly $100 \%$ Exciton Use and Negligible Efficiency Roll-Off. Angew. Chem. Int. Ed. 57, 9290-9294 (2018). 
4. Ohisa, S. et al. An Indolocarbazole-Based Thermally Activated Delayed Fluorescence Host for Solution-Processed Phosphorescent Tandem Organic Light-Emitting Devices Exhibiting Extremely Small Efficiency Roll-Off. Adv. Funct. Mater., 1808022 (2019).

5. Wu, Z.G. et al. Chiral Octahydro-Binaphthol Compound-Based Thermally Activated Delayed Fluorescence Materials for Circularly Polarized Electroluminescence with Superior EQE of 32.6\% and Extremely Low Efficiency Roll-Off. Adv. Mater., 1900524 (2019).

6. Yang, Y. et al. Thermally Activated Delayed Fluorescence Conjugated Polymers with Backbone-Donor/Pendant-Acceptor Architecture for Nondoped OLEDs with High External Quantum Efficiency and Low Roll-Off. Adv. Funct. Mater. 28, 1706916 (2018).

7. Wang, Z. et al. Exciton-Adjustable Interlayers for High Efficiency, Low Efficiency RollOff, and Lifetime Improved Warm White Organic Light-Emitting Diodes (WOLEDs) Based on a Delayed Fluorescence Assistant Host. Adv. Funct. Mater. 28, 1706922 (2018).

8. Kotadiya, N.B. et al. Efficient and stable single-layer organic light-emitting diodes based on thermally activated delayed fluorescence. Nat. Photonics 13, 765-769 (2019).

9. Murawski, C. et al. Efficiency roll-off in organic light-emitting diodes. Adv. Mater. 25, 6801-6827 (2013).

10. Giebink, N.C. et al. Quantum efficiency roll-off at high brightness in fluorescent and phosphorescent organic light emitting diodes. Phys. Rev. B 77, 235215 (2008).

11. Brédas, J.-L. et al. Organic semiconductors: A theoretical characterization of the basic parameters governing charge transport. Proc. Natl. Acad. Sci. 99, 5804-5809 (2002).

12. Cui, L.-S. et al. Long-lived efficient delayed fluorescence organic light-emitting diodes using n-type hosts. Nat. Commun. 8, 2250 (2017).

13. Hassine, L. et al. Transient response of a bilayer organic light emitting diode: Building-up of external and recombination currents. J. Appl. Phys. 91, 5170-5175 (2002).

14. Aziz, Hany, et al. Degradation mechanism of small molecule-based organic light-emitting devices. Science 283, 1900-1902 (1999). 
15. Obolda, A. et al. Triplet-Polaron-Interaction-Induced Upconversion from Triplet to Singlet: a Possible Way to Obtain Highly Efficient OLEDs. Adv. Mater. 28, 4740-4746 (2016). 16. Zhang, Y. et al. Simultaneous enhancement of efficiency and stability of OLEDs with thermally activated delayed fluorescence materials by modifying carbazoles with peripheral groups. Sci. China Chem. 62, 393-402 (2019).

17. Zhang, Q. et al. Efficient blue organic light-emitting diodes employing thermally activated delayed fluorescence. Nat. Photonics 8, 326 (2014).

18. Du, M. et al. Novel Emitting System Based on a Multifunctional Bipolar Phosphor: An Effective Approach for Highly Efficient Warm-White Light-Emitting Devices with High Color-Rendering Index at High Luminance. Adv. Mater. 28, 5963-5968 (2016).

19. Miao, Y. et al. Precise manipulation of the carrier recombination zone: a universal novel device structure for highly efficient monochrome and white phosphorescent organic lightemitting diodes with extremely small efficiency roll-off. J. Mater. Chem. C 6, 8122-8134 (2018).

20. Einzinger, M. et al. Shorter Exciton Lifetimes via an External Heavy-Atom Effect: Alleviating the Effects of Bimolecular Processes in Organic Light-Emitting Diodes. Adv. Mater. 29, 1701987 (2017).

21. Zhao, F. et al. Spatial exciton allocation strategy with reduced energy loss for highefficiency fluorescent/phosphorescent hybrid white organic light-emitting diodes. Mater. Horiz. 4, 641-648 (2017)

22. Song, W. et al. Host Engineering for High Quantum Efficiency Blue and White Fluorescent Organic Light-Emitting Diodes. Adv. Mater. 27, 4358-4363 (2015).

23. Li, X. et al. Multiphotoluminescence from a Triphenylamine Derivative and Its Application in White Organic Light-Emitting Diodes Based on a Single Emissive Layer. Adv. Mater., 1900613 (2019) 
24. Lee, J. et al. Hot excited state management for long-lived blue phosphorescent organic light-emitting diodes. Nat. Commun. 8, 15566 (2017).

25. Kotadiya, N.B. et al. Universal strategy for Ohmic hole injection into organic semiconductors with high ionization energies. Nat. Mater. 17, 329 (2018).

26. Liu, B.-Q. et al. Extremely high-efficiency and ultrasimplified hybrid white organic lightemitting diodes exploiting double multifunctional blue emitting layers. Light Sci. Appl. 5, e16137 (2016).

27. Li, Y. et al. Tailor-made nanostructures bridging chaos and order for highly efficient white organic light-emitting diodes. Nat. Commun. 10, 2972 (2019).

28. Berger, L. Exchange interaction between ferromagnetic domain wall and electric current in very thin metallic films. J. Appl. Phys. 55, 1954-1956 (1984).

29. Slowik, I. et al. New concept for organic light-emitting devices under high excitations using emission from a metal-free area, Vol. 9895. (SPIE, 2016).

30. Slowik, I. et al. Novel organic light-emitting diode design for future lasing applications. Org. Electron. 48, 132-137 (2017).

31. Burlingame, Q. et al. Centimetre-scale electron diffusion in photoactive organic heterostructures. Nature 554, 77 (2018).

32. Coburn, C. et al. Organic Charge-Coupled Device. ACS Photonics 6, 2090-2095 (2019).

33. Ou, Q.D. et al. Extremely Efficient White Organic Light-Emitting Diodes for General Lighting. Adv. Funct. Mater. 24, 7249-7256 (2014).

34. Wu, Z. et al. Strategic-tuning of radiative excitons for efficient and stable fluorescent white organic light-emitting diodes. Nat. Commun. 10, 2380 (2019).

35. Tang, X. et al. High-Quality White Organic Light-Emitting Diodes Composed of Binary Emitters with Color Rendering Index Exceeding 80 by Utilizing Color Remedy Strategy. $A d v$. Funct. Mater. 29, 1807541 (2019). 
36. Wu, S. F. et al. White Organic LED with a Luminous Efficacy Exceeding $100 \mathrm{~lm} \mathrm{~W}^{-1}$ without Light Out-Coupling Enhancement Techniques. Adv. Funct. Mater. 27, 1701314 (2017). 37. Hassine, L. et al. Transient response of a bilayer organic electroluminescent diode: Experimental and theoretical study of electroluminescence onset. Appl. Phys. Lett. 78, 10531055 (2001).

38. Volkov, A. V. et al. Understanding the capacitance of PEDOT: PSS. Adv. Funct. Mater. 27, 1700329 (2017).

39. Alemu, D. et al. Highly conductive PEDOT: PSS electrode by simple film treatment with methanol for ITO-free polymer solar cells. Energ. Environ. Sci. 5, 9662-9671 (2012).

40. Lasia, A. Electrochemical impedance spectroscopy and its applications. In Modern aspects of electrochemistry, pp. 143-248. Springer, Boston, MA, 2002.

41. Lee, J. H. et al. Langevin and Trap-Assisted Recombination in Phosphorescent Organic Light Emitting Diodes. Adv. Funct. Mater. 24, 4681-4688 (2014).

\section{Acknowledgements}

This work was supported by the National Natural Science Foundation of China (Grant Nos. 61774074, 61905086), Science and Technology Development Planning of Jilin Province (Project Nos. 20190101024JH, 20200201296JC), the Hong Kong Scholars Program (Project No. XJ2020028) and grants from the Research Grants Council of the Hong Kong Special Administrative Region, China (Project Nos. 11300418 and 11300419). Thank Dr. Jinsong Huang from Oxford Suzhou Centre for Advanced Research (OSCAR) for his constructive suggestions.

\section{Author contributions}

W. F. X. conceived the central ideas and concepts. W. F. X. and C. S. L. supervised the work. W. F. X., C. S. L. and S. H. L. designed and conducted the whole experiments, analyzed the data and prepared the manuscript. X. Z. designed the ITO grid array anode, fabricated the blue, red phosphorescent devices and TADF devices, and analyzed the data. H. W. Y performed the 
device lifetime measurements. C. X. Z. measured the transient voltage and current characteristics, collected the infrared images and analyzed the data. L. T. Z. performed the transient EL response measurements and analyzed the data. D. S. and M. F. L. measured and analyzed the XPS spectra. M. L. L. conducted the impedance spectra measurements, and analyzed the data. W. F. X., C. S. L. and S. H. L. conducted the theoretical analysis and calculations. H. W. and Z. Q. T. gave their support for the theoretical analysis about Warburg element. All authors discussed the results and assisted in the data interpretation and manuscript preparation.

\section{Additional Information}

Methods, Figures S1-S23, Table S1-3, Supplementary Notes 1-6 and Equations S1-S16 are available from Supporting Information.

Conflict of Interest: The authors declare no conflict of interest. 
$\mathbf{a}$

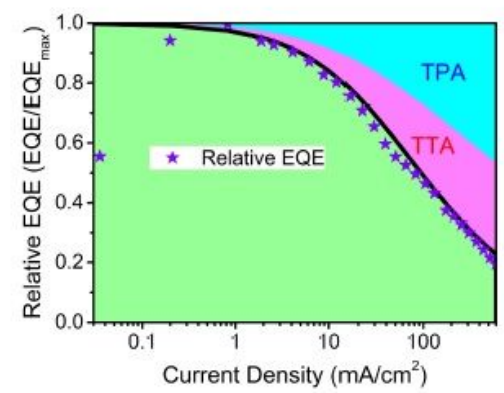

d

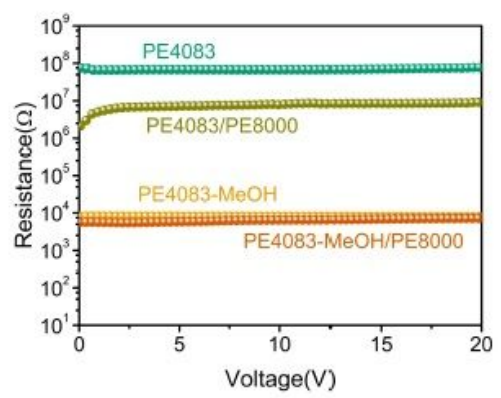

b

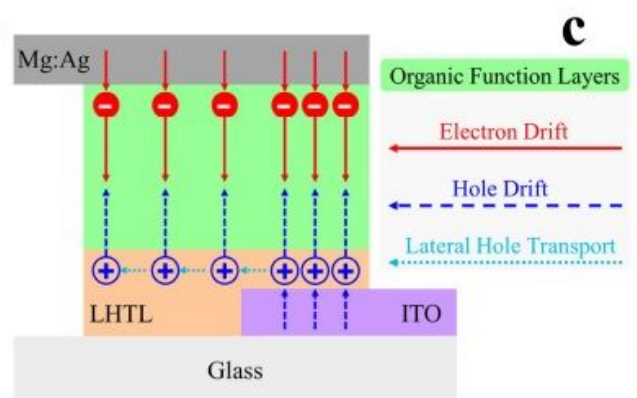

$\mathbf{e}$

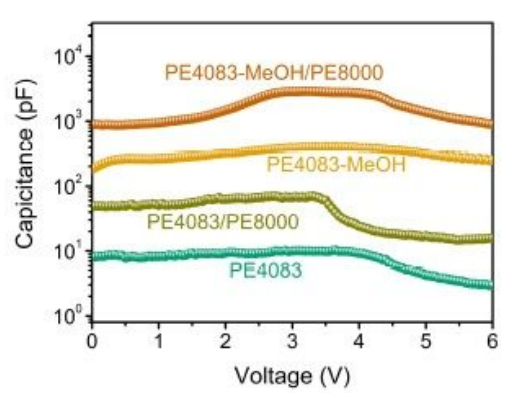

$\mathbf{f}$

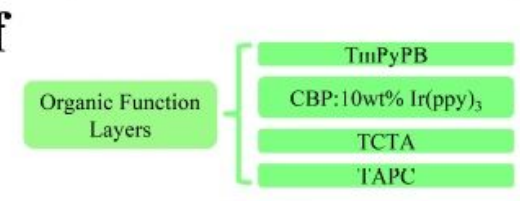

LHTL

\begin{tabular}{|c|c|c|c|}
\hline \multirow[t]{2}{*}{ PE4083 } & PE8000 & PE4083 & PE8000 \\
\hline & PE4083 & $-\mathrm{MeOH}$ & $\begin{array}{l}\text { PE4083 } \\
-\mathrm{MeOH}\end{array}$ \\
\hline Device C1 & Device C2 & Device D1 & Device D2 \\
\hline
\end{tabular}

g
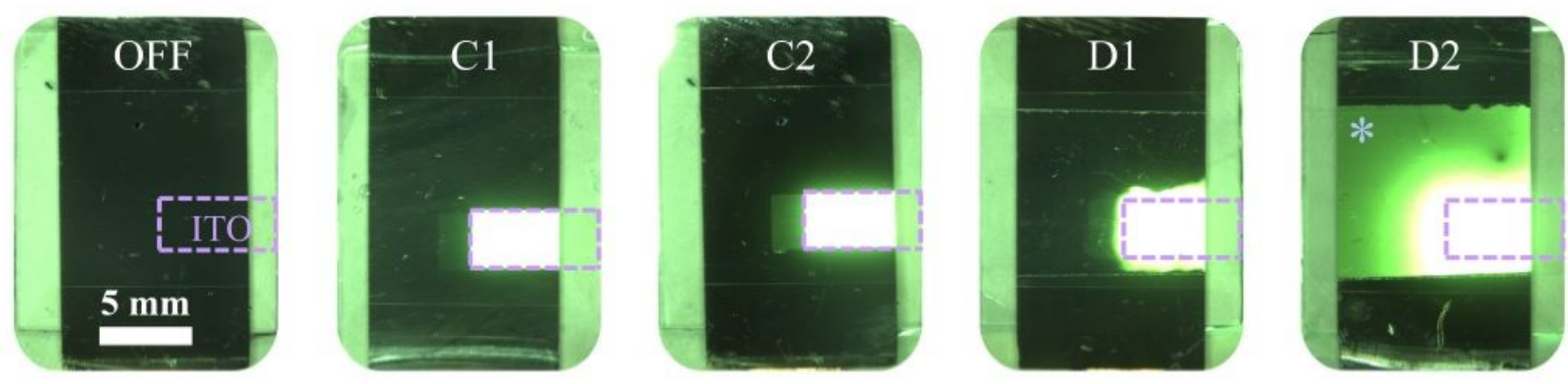

\section{Figure 1}

(a) Theoretically calculated contributions of TTA and TPA to EQE loss in device C1 as a function of current density. Experimentally measured relative EQE is shown as symbol *; (b) Side-view schematic diagram of an OLED with a lateral-hole-transport layer, and (c) its top view. (d) Lateral resistance and (e) capacitance characteristics of different types of PEDOT:PSS layers. (f) Devices structures of devices C1, C2, D1 and D2. (g) Top-view images of these devices under $6 \mathrm{~V}$. 
a

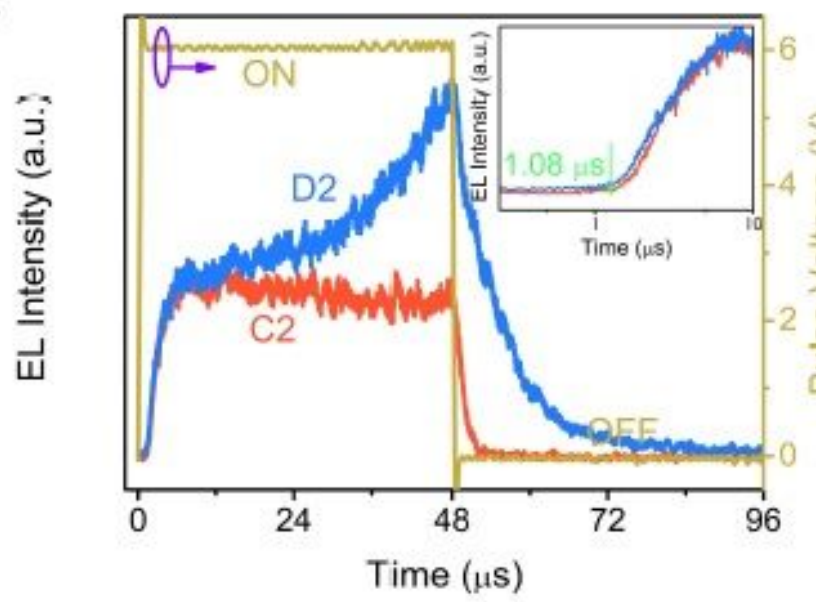

c

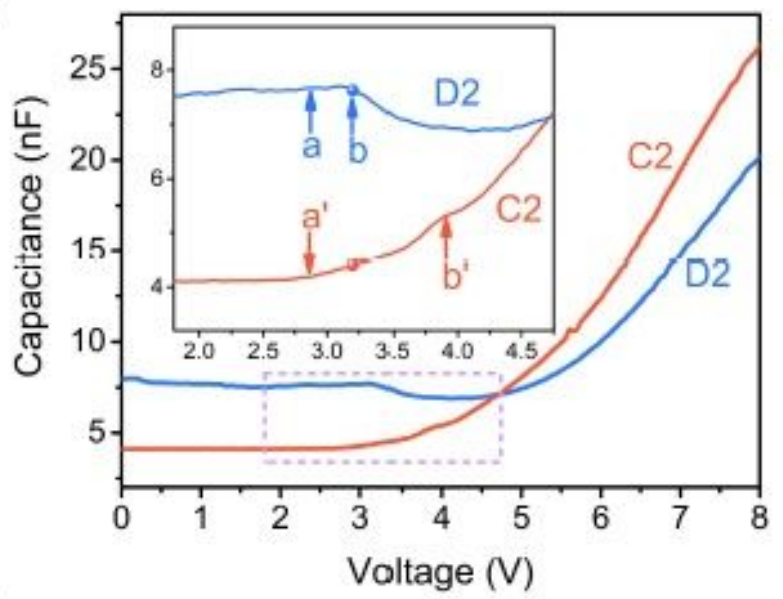

e

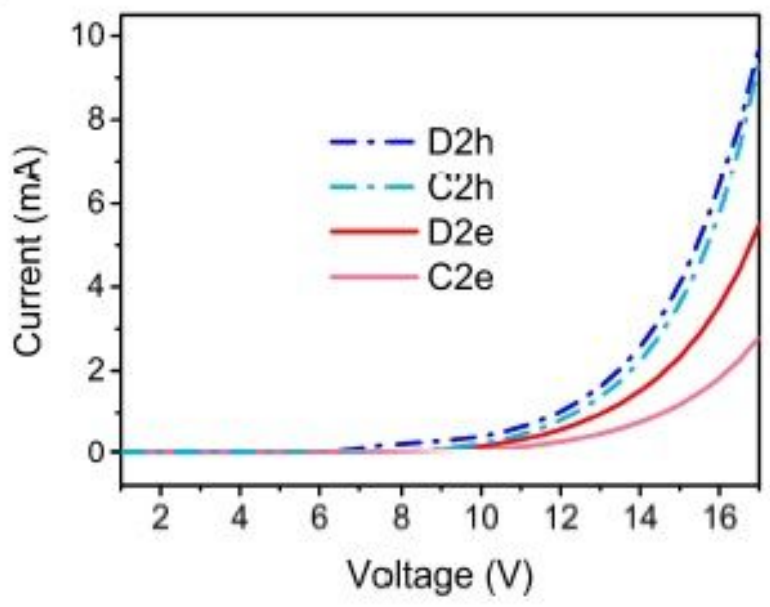

b

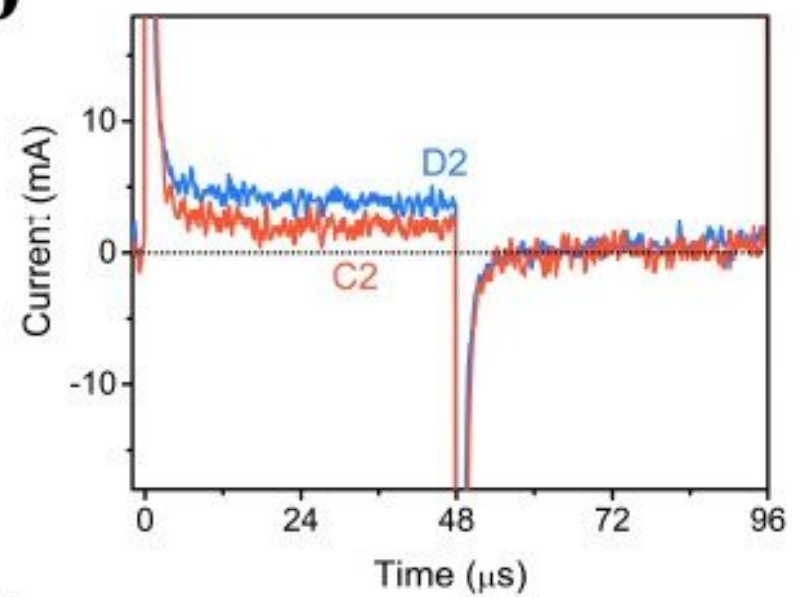

d

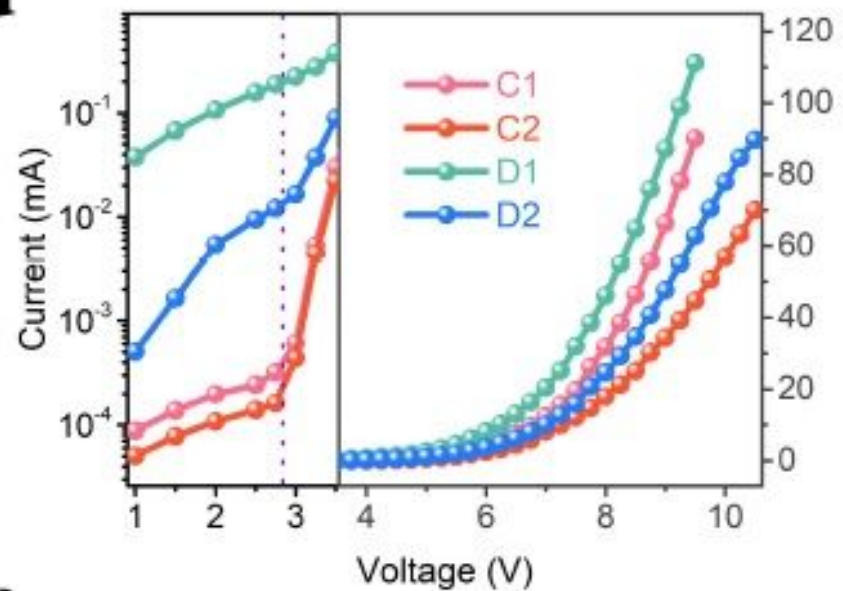

f

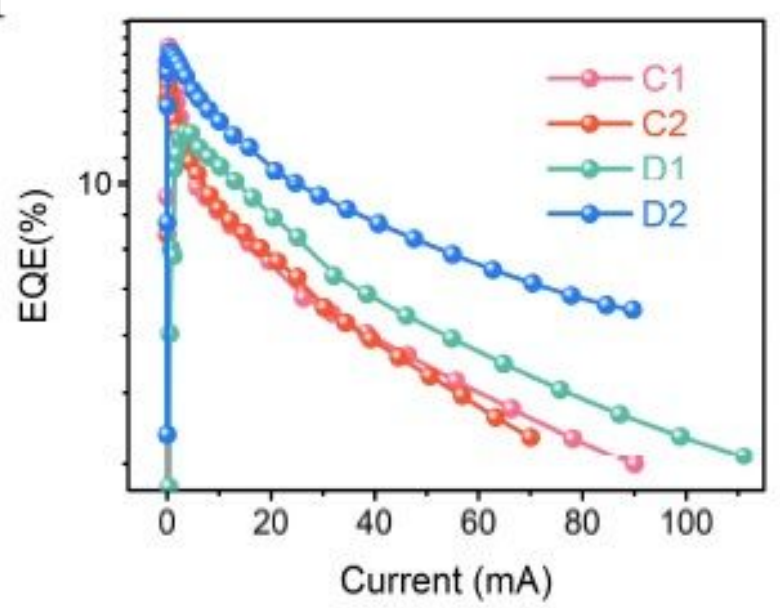

Figure 2

(a) Transient EL responses of devices C2 and D2, and (b) their transient current characteristics; the voltage pulse width and heights were respectively $48 \mu$ s and 6 V. (c) Capacitance-voltage characteristics of devices C2 and D2. (d) Current-voltage characteristics of devices C1, C2, D1 and D2. (e) Current-voltage characteristics of single carrier devices for device C2 and D2 (-e and -h represents electron-only device and hole-only device, respectively). (f) EQE-current characteristics of devices C1, C2, D1 and D2. 
Working mechanism of an OLED with a LHDL

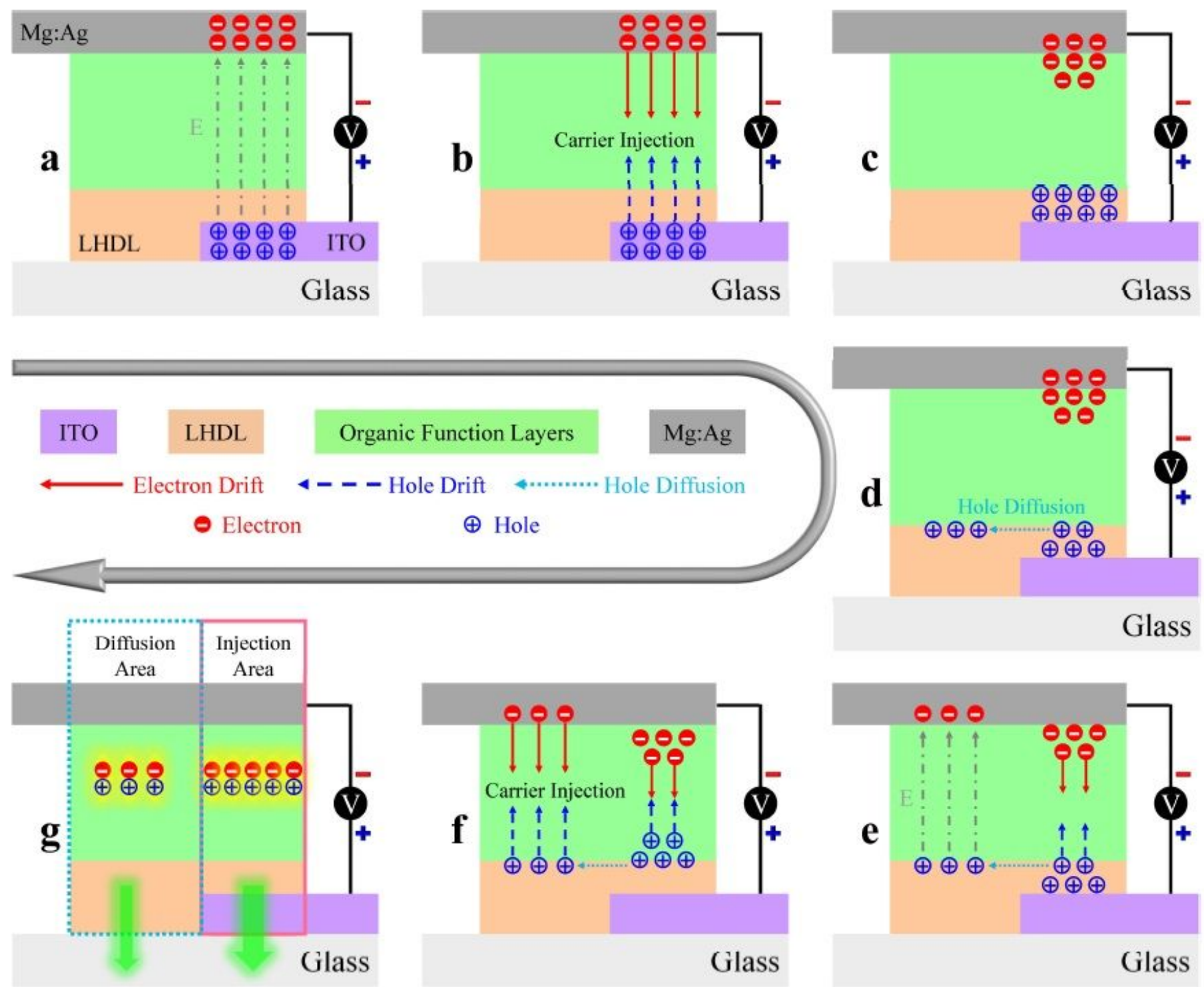

\section{Figure 3}

a working mechanism of an OLED with lateral hole diffusion. (a) Applying bias voltage across the anode and cathode. (b) Carrier injection in injection area. (c) Asymmetric hole and electron transport abilities. (d) Holes lateral diffusion. (e) Electrical field formation in diffusion area (i.e. area outside ITO-cathode overlapping region). (f) Carrier redistribution and injection in diffusion area. (g) Exciton recombination radiation. 
$\mathbf{a}$

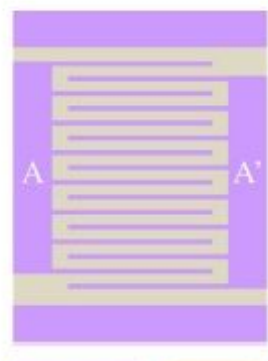

\section{ITO}

$\mathrm{Mg}: \mathrm{Ag}$
LHDL

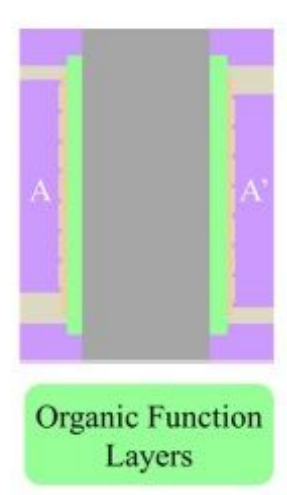

b

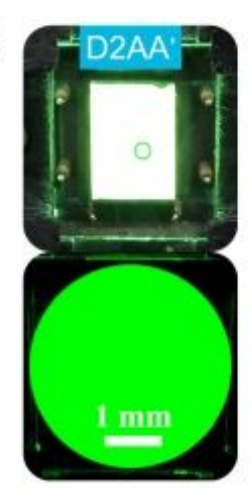

TmPyPB

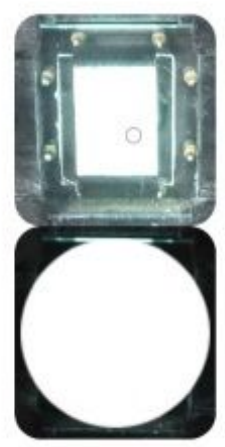

Organic Function Layers

TCTA : $10 w t \%$ Firpic

TCTA

TAPC

Lateral Diffusion Layer

PE8000

PE4083

Device WCAA'
PE8000

PE4083-MeOH

Device WDAA'
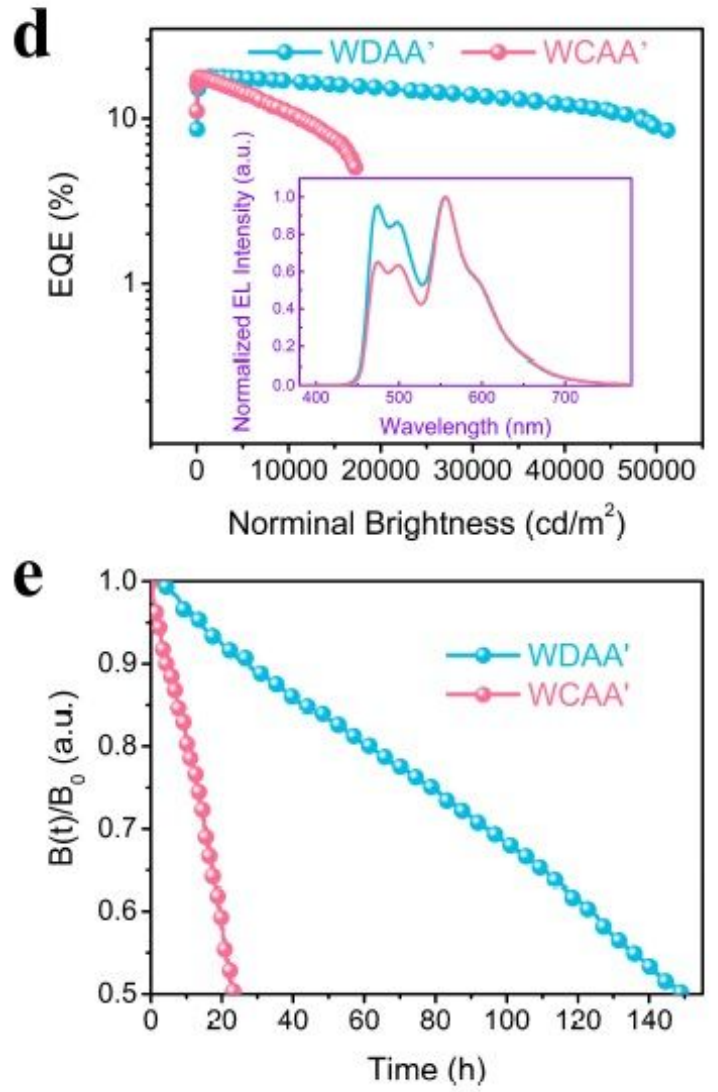

Figure 4

(a) Schematic diagram of the ITO grid array anode and the corresponding OLED. (b) Photographs (upper row) of operating device D2AA' with the ITO grid array anodes. Lower one shows magnified images from the green circles marked in the upper picture. (c) Photographs of white device WDAA' and structure details of white device WDAA' and WCAA'. (d) EQE-nominal brightness characteristics of the two white devices. Inset shows their EL spectra at $25 \mathrm{~mA} / \mathrm{cm} 2$. (e) Normalized brightness degradation $B(t) / B 0(B 0=1000$ $\mathrm{cd} / \mathrm{m} 2)$ of white devices WDAA' and WCAA'.

\section{Supplementary Files}

This is a list of supplementary files associated with this preprint. Click to download.

- Supportinglnformation.pdf 